\title{
Télévision par satellite et liaisons télématiques dans l'enseignement de l'anglais de spécialité, résultats et perspectives
}

Hervé Thily

\section{(2) OpenEdition}

\section{Journals}

Édition électronique

URL : http://journals.openedition.org/asp/4391

DOI : 10.4000/asp.4391

ISSN : 2108-6354

\section{Éditeur}

Groupe d'étude et de recherche en anglais de spécialité

Édition imprimée

Date de publication : 1 mars 1993

Pagination : 437-447

ISSN : 1246-8185

\section{Référence électronique}

Hervé Thily, «Télévision par satellite et liaisons télématiques dans l'enseignement de l'anglais de spécialité, résultats et perspectives », ASp [En ligne], 1 | 1993, mis en ligne le 03 juin 2014, consulté le 02 mai 2019. URL : http://journals.openedition.org/asp/4391 ; DOI : 10.4000/asp.4391

Ce document a été généré automatiquement le 2 mai 2019.

Tous droits réservés 


\title{
Télévision par satellite et liaisons télématiques dans l'enseignement de l'anglais de spécialité, résultats et perspectives
}

\author{
Hervé Thily
}

1 L'antenne parabolique pour la réception des TV satellitaires est un élément de plus en plus familier sur les superstructures des établissements. Paradoxalement, le potentiel pédagogique de ce dernier venu dans le monde des hypermédias reste encore très mal connu, voire totalement ignoré de nombreux enseignants. Un autre outil de travail, la télématique, souffre aussi de sa haute technicité et de ce fait demeure malheureusement le fait d'initiés, car perçue comme étant l'aboutissement d'une longue et laborieuse formation informatique.

2 Il serait malhonnête de nier pour l'utilisateur la nécessité d'un apprentissage axé essentiellement, dans un premier temps, sur la manipulation des divers matériels regroupés sous le vocable «technologies nouvelles». Il serait tout aussi stupide de se priver d'une véritable manne de ressources (images vidéo et données informatiques de tous ordres). À brève échéance, comme le montrent les diverses expérimentations menées par la Direction des Lycées et Collèges au Ministère de l'Éducation nationale, c'est la didactique et la méthodologie qui sont appelées à connaître un profond renouveau.

Les bouleversements technologiques qui sont intervenus ces dernières années ne peuvent expliquer à eux seuls l'émergence de nouvelles formes d'apprentissage et leur succès. Les facteurs économiques et géopolitiques dans une Europe en mutation ont largement contribué à la mise en place des premières structures d'une synergie supranationale, à très haut niveau, des ressources humaines et matérielles dans les domaines de la formation, qu'elle soit initiale ou continue, en milieu scolaire ou professionnel. Si l'enseignant n'a bien évidemment que très peu d'influence sur le politique ou l'économique, il lui appartient cependant de mettre à profit, avec un opportunisme de 
tous les instants, la profusion de documents véhiculés par les technologies nouvelles pour, à terme, espérer influer sur le contenu de ces dernières.

4 L'enseignant de langue de spécialité peut trouver, grâce aux satellites et la télématique, diverses réponses à ses besoins en documents authentiques. Pour plus de commodité, je renverrai aux définitions de ce terme proposées par H. Holec (1991) et M. Perrin (1990).

5 Examinons en premier lieu les ressources propres aux TV satellitaires actuelles.

\section{Les chaînes de l'espace}

6 C'est à dessein que j'emprunte ce terme à Philippe Gassner pour désigner un médium qui ne diffère guère des TV hertziennes que par son mode de transmission. Par delà les normes de diffusion (PAL, SECAM et D2MACPAQUET), le mode de réception (cryptage ou diffusion en clair), câble ou en réception directe, ainsi que les divers types de satellites en orbite géostationnaire, il convient de distinguer deux conceptions radicalement différentes en matière de TV satellitaire.

7 Le premier groupe de chaînes de l'espace - de loin le plus important - coiffe toutes les diffusions destinées au "grand public européen ». Les thématiques tournent autour du divertissement ou de l'information. Le guide pratique d'utilisation récemment publié par ATENA/Montpellier est, à cet effet, un excellent outil d'exploration et suggère diverses applications pédagogiques possibles au regard des programmations. Cette source de documents, où l'anglais tient une part prépondérante, est d'une grande richesse, car en constante expansion. Zsuzsanna Ardo, au dernier Congrès mondial de la FIPLV en Hongrie, indiquait que les chaînes de l'espace allaient voir leur nombre tripler en cinq ans!

8 C'est dans cette catégorie que je trouve depuis 1988 les extraits vidéo utilisés en classes de Techniciens supérieurs Conception de Produits Industriels et Productique. La série australienne Beyond 2000 - très proche de son homologue Tomorrow's World de la $\mathrm{BBC}-$ m'apporte régulièrement des extraits pour appuyer mon enseignement en anglais de spécialité dans ces sections. Pour intégrer ces documents dans une séance vide venant en point d'orgue d'une unité thématique et fonctionnelle cohérente, diverses aides méthodologiques, visuelles ou informatiques sont ensuite mises au point, le plus souvent avec les étudiants et les collègues des disciplines scientifiques et techniques de ces sections.

9 Le second groupe, bien évidemment minoritaire, recouvre les chaînes à vocation didactique, pour divers publics :

- Canal Santé, pour les professions médicales

- Channel E pour l'ouverture sur l'Europe, en multilangues,

- Computer Channel (Informatique et Télécom Technologies)

- Europace (Haute Technologie - Enseignement supérieur)

- Eurostep, en multilangues, sur le satellite OLYMPUS ainsi que

- RAISAT « educational » conjointement sur le canal 24 depuis quelques mois.

On citera aussi BBC World Service, et la Sept. Les cours réalisés par le CNED en VTI (Video Transmission Interactive) sur EUTELSAT 2 VF 1 touchent un public de plus en plus vaste. Les besoins en formation post-baccalauréat sont tels que l'EAD ou Enseignement à distance ne peut que se développer. Le hasard veut qu'au moment où je vous parle, 
ATENA organise précisément au Futuroscope de Poitiers une journée d'étude exceptionnelle sur ce thème, en liaison avec l'Université de Stanford.

Dans le cadre de l'expérimentation du DBS OLYMPUS menée depuis 1990 par la DLC15, il a été possible de trouver pour le Génie civil, les techniques chirurgicales, les vétérinaires, les architectes, l'électronique et l'informatique des émissions traitant des techniques de pointe dans ces professions et quelquefois diffusés en multilangues. Terry Brockley conçoit même des vidéothèques interactives et spécifiques, enrichies en permanence par les diffusions venues de l'espace.

12 Pour l'enseignant en langue de spécialité, qui recherche des documents vidéos s'inscrivant dans la pratique professionnelle, le problème relève plus de l'adéquation des extraits que de leur nombre. Le gros travail préparatoire de pédagogisation, que John McGovern, en LGP, fixait à 40 pour 1, fait de la sélection des vidéos une étape-clef dans la stratégie didactique. Les contraintes horaires et les objectifs de la formation conditionnent le choix des apprentissages et leurs supports.

Dans ce contexte, les chaînes de l'espace, éducatives ou non, offrent, pour un travail en différé, les avantages suivants :

- authenticité linguistique, sans ST ou doublage.

- grand intérêt professionnel

- pertinence et actualité des contenus

- valorisation de la perception de la langue de spécialité.

\section{Télématique}

La télématique dans ses applications pédagogiques, actuellement expérimentée dans le cadre d'EDU2000 par la DLC15, vient, dans mon établissement de ROUEN, compléter et renforcer le dispositif déjà en place constitué par le réseau des viewing sites européens, axés sur OLYMPUS ou ceux, plus nombreux, pointés sur les deux ASTRA actuels (1A et 1B). Si EUROSTEP/OLYMPUS sur son canal d'origine permettait le télétexte, la spécificité de la norme D2MAC, les interrogations qu'elle soulève, ont freiné la diffusion de ce satellite résolument d'avant-garde, tant du point de vue technique que pédagogique.

15 À l'heure actuelle, l'instauration de passerelles entre les deux réseaux télématiques à vocation pédagogique, EDUTEL pour la France et CAMPUS 2000 pour la Grande-Bretagne permet d'accéder à diverses banques de données ou d'instaurer une interactivité originale entre établissements connectés au réseau. Les Britanniques ont déjà réussi, en LGP, des opérations combinées faisant appel tour à tour à la TV satellite et à la télématique. Le projet GEMINI, de Ian Webster ainsi que le European Studies Project de Roger Austin, entre autres, trouvent dans les Technologies nouvelles des possibilités d'expression, simples, motivantes et d'une grande efficacité.

Que peut trouver l'enseignant en langue de spécialité dans les réseaux télématiques couverts par EDU2000? Des réponses immédiates, fiables et appropriées à divers problèmes liés à la recherche documentaire et aux applications pédagogiques. Cet outil permet aux enseignants et étudiants de sortir enfin du traditionnel face-à-face confiné à la salle de classe. La communication en simulation, de règle jusqu'alors, fait place à l'authentique, à la communication en situation, rendue possible grâce à la télématique (ou les liaisons en phonie). Voici quelques pistes possibles, parmi bien d'autres, qui ne demandent qu'à être explorées. 


\subsection{Les banques de données en ligne} industriels, mais accessibles sur les réseaux français, britanniques et nord-américains. Elles ouvrent un champ très vaste de possibilités et constituent déjà une forme de communication pour :

- rechercher des stages professionnels à l'étranger

- prospecter des établissements en vue de compléter sa formation à un niveau Bac+4 (Polytechnics, par exemple).

- faire le montage financier en vue d'un voyage d'études

- obtenir de la documentation scientifique et technique (auprès d'organismes ou établissements de formation)

- connaitre les articles publiés dans le New Scientist avec une recherche par mots-clef (60p/ minute sur PROFILE).

- connaitre à l'avance le contenu précis des émissions diffusées en clair sur les chaînes SKY (BDD CAMPUS SATELLITE EDUCATION PROJECT DATABASE), comme, par exemple les reportages de la série BEYOND 2000. À l'heure actuelle, ces informations ne sont publiées que sur le seul territoire du Royaume-Uni.

\subsection{La messagerie et la téléconférence}

La messagerie permet l'échange de messages entre établissements sur des préoccupations ou attentes communes. Nous envisageons une correspondance entre notre Lycée Technique et une Polytechnic dispensant les mêmes formations (Productique, Conception de Produits Industriels).

Une téléconférence participe d'un principe analogue mais se caractérise par un accès beaucoup plus ouvert sur un ou plusieurs thèmes préalablement définis et correctement préparés. Dans notre esprit, cette forme de communication, en langue de spécialité, peut parfaitement venir compléter, en temps limité, l'apport de la TV satellitaire.

\subsection{La conjonction des technologies nouvelles}

Dès la rentrée de septembre, les Techniciens supérieurs du Lycée Blaise Pascal de Rouen se verront présenter toute une unité thématique (Tomorrow's Trains) de cours d'anglais de spécialité faisant appel, à tous les stades, aux technologies nouvelles. Cette unité de cours n'est rendue possible que grâce à l'appui et la collaboration des responsables britanniques (SKY et BT/CAMPUS 2000) et australiens (le Groupe BEYOND). Elle aura pour objet de solliciter les quatre compétences et de valider par une dynamique du succès les acquis des apprenants placés systématiquement en situation de communication authentique.

Le support écrit sera un article spécialisé, tiré de New Scientist, préalablement sélectionné dans la Banque de données PROFILE. Les autres articles capturés par le mot-clef mais non sélectionnés pour le travail en cours seront regroupés dans une banque de données d'accès facile. Tous les éléments lexicaux du texte retenu auront été répertoriés et entrés, après vérification auprès des collègues et/ou industriels dans une banque de données trilingue regroupant les termes techniques industriels rencontrés dans la spécialité. Cette BDD a été une conception collective au Lycée sur un appui logistique en DBASE III à 
l'usage des étudiants et enseignants. Il est prévu qu'à terme cette base de données soit consultable sur 3614 EDUTEL, devenant de ce fait accessible, à moindre coût, de tout point du territoire national et de l'étranger.

La séquence vidéo aura été captée par TV satellite et sera étudiée en différé. Des aides visuelles (tablette/rétroprojecteur + transparents classiques), des schémas et des procédés mnémoniques pour le réemploi des structures syntaxiques récurrentes seront mis au point en vue de l'élucidation collective et conviviale des contenus visuel et sonore du document. Si un établissement britannique, ou plusieurs, s'associe(nt) à ce projet, nous établirons alors un débat télématique avec plusieurs objectifs :

- valider la compréhension du document visionné.

- analyser et commenter les choix scientifiques et techniques retenus par les concepteurs.

- échanger impressions ou critiques entre étudiants et/ou enseignants

- donner des informations et références sur des prototypes, matériaux et approches analogues ou concurrentes, etc.

À tout moment, tout comme les étudiants du Lycée Blaise Pascal de Rouen, les interlocuteurs britanniques (ou d'ailleurs) pourront consulter la banque de données de termes techniques industriels, si elle se trouve sur serveur MINITEL à cette date.

Les résultats observés depuis maintenant près de quatre ans indiquent que, grâce à un recours croissant aux technologies nouvelles, la langue de spécialité est davantage perçue par les apprenants comme étant partie intégrante d'une formation professionnelle de qualité. Mieux acceptée, elle accompagne les innovations scientifiques et technologiques par lesquelles elle nous parvient. C'est en retrouvant son terrain originel, celui de la communication dans et pour le monde du travail, que la LSP renoue avec sa véritable fonction. Dans cette optique, les technologies nouvelles, qu'elles soient satellitaires ou télématiques, ouvrent ainsi des perspectives prometteuses pour une interactivité très motivante et un partage des connaissances sans précédent.

\section{BIBLIOGRAPHIE}

Ardo, Z. 1991. « The European Space Agency (ESA), OLYMPUS and Tele-Education ».

Communication Symposium Enseignement des Langues par Satellites XVII Congrès mondial FIPLV Pécs, août 1991.

Austin, R. 1991. « Satellite and electronic mail ». The Eurostep Courier June, 16-17.

Brockley, T. 1991. « An Exercise in networking schools satellite broadcasting ». The Eurostep Courier May, 6-11.

Coleman J. 1990. « Starting with satellite: A basic guide to using off-air video recordings in the language classroom ». Language Learning Journal Sept., 16-18.

Coulardeau, J. 1991. « Interactivité, câble et formation à distance ». In Actes XII Colloque du GERAS février 1991, 67-73. 
Holec, H. 1991. « Des documents authentiques, pour quoi faire? ». In Stratégies pédagogiques et outils pour l'enseignement des langues étrangères. Dijon : C.R.D, 19-31.

McGovern, John. 1983. « Types of video software: A user's experience ». In Video Applications in English Language Teaching ELT Documents 114. Londres : Pergamon Press/the British Council.

Perrin, M. 1990. « De l'utilisation 'communicative' des documents authentiques ». In Actes XI Colloque du GERAS avril 1990, 9-34.

Stenton, A. J. 1991. « Education as a prerequisite for independence ». In Actes XII Colloque du GERAS février 1991, 298-329.

Thily, H. 1991. « Satellite TVs and prospects for ELT ». Communication au Symposium de l'enseignement des langues par satellites XVII Congrès mondial FIPLV Pécs Hongrie août 1991.

Thily, H. 1992. « Le voir et le dire ou l'expression du point de vue ». In Annales de l'Université de Savoie 15, 151-163.

\section{ANNEXES}

\section{Annexes : adresses et rapports utiles}

ATENA - Des Satellites pour l'éducation et la formation, Guide pratique d'utilisation 1991. Association des utilisateurs de technologies éducatives pour de nouveaux apprentissages (ATENA), BP 1107 - 34007 Montpellier Cédex 1. Tél. 676089 42, Fax 67606119.

BSB - British Sky Broadcasting Ltd, 6 Centaurs Business Park Grant way, Isleworth, Middlesex TW7 5QD, Royaume-Uni. Tél. 071782 3000, Fax 0717823113.

CAMPUS 2000 - Contact Eva Barowska, Priory House St John's Lane, Londres EC1M 4HD, Royaume-Uni.

CNDP - Étude menée par P. Gasser, « Les chaînes de l'espace », avril 1990.

Direction des Lycées et Collèges DLC 15 - Nouvelles Technologies - ministère de l'Éducation nationale, 107 rue de Grenelle, 75007 - PARIS - Langues et Télévisions par Satellite Langues Vivantes et Technologies Nouvelles, Dijon nov. 1990

EUROPACE - CNIT-INFORMAT, Boîte Privée 529, 2 place de la Défense, 92053 Paris La Défense.

EUROSTEP/Olympus, PO Box 11112, 2301 EC Leiden Pays-Bas. Tél. +31 71 120863, Fax +31 71134545 .

FIPLV - XVII Congrès mondial août 1991 Pécs. Dr Simon Ibolya Kakas, Université de Pecs, 7636 Pecs, Hongrie.

SATELLITE TV EUROPE - Les Programmes de la TV par satellite, 17 quai Louis XVIII, 33000 Bordeaux. Tél. 565104 17, Fax 56811084.

SOBA - School Olympus Broadcast Association, Gwynedd TVEI Unit - Technology Centre, Bridge St., Llangefni, Anglesey LL77 7TW Royaume-Uni. Tél. (0)248 724929, Fax (0)248 724674. 


\section{AUTEUR}

\section{HERVÉ THILY}

Enseignant en STS, chargé d'expérimentation DLC 15, Lycée Blaise Pascal Technique, Rouen. herve.thily@univ-rouen.fr 